\title{
Desenvolvimento e validação de um questionário sobre uso do Facebook voltado para praticantes de atividades físicas em academias
}

\section{Development and validation of a questionnaire on Facebook use to gym users}

\author{
Matheus Pedroso'; Rodrigo Sartori²; Caroline Pietta Dias³; Cristian Roncada ${ }^{4}$
}

\section{Resumo}

A prática de atividades físicas vem sofrendo modificações com o avanço da internet. Usuários de academias utilizam desta tecnologia para se conectarem as redes sociais virtuais como o Facebook. Assim, o estudo tem como objetivo construir e validar um questionário sobre o uso do Facebook e a prática de atividades físicas. A partir do referencial teórico elaborou-se 20 itens separados em cinco domínios, com respostas em escala do tipo Likert, com pontuação final de 0 a 100. A validação interna do instrumento foi feita em cinco etapas: a) alfa de Cronbach $(\alpha C)$; b) Correlação de Kendall; c) coeficiente de correlação intraclasse; d) efeitos de teto/piso; e) índice de Kappa. A amostra foi composta por 385 alunos de academias, sendo 273 do sexo feminino (70,9\%), com média de idade $31,61 \pm 9,62$ anos. Os valores atribuídos ao coeficiente de $\alpha \mathrm{C}$ demonstram haver excelente fiabilidade entre perguntas (itens) e suas respostas $(\alpha C=0,923)$. Ao aplicarmos a correlação do $\alpha \mathrm{C}\left(\mathrm{r}^{2}\right)$ encontramos todos os valores acima do esperado: $\geq 0,3$, reforçando uma associação positiva entre itens e categorias. Os valores encontrados com a aplicação dos efeitos do teto/piso nos mostram que 10 itens (50\%) apresentaram um efeito piso. A reprodutibilidade por meio do índice de Kappa atribuiu valor máximo (muito bom) para 11 itens, sendo que nenhum ficou na classificação mais fraca (pobre). Por fim, a validação demonstrou que o instrumento possui um bom desempenho para avaliar o que se propõe.

\section{Palavras-chave}

Inquéritos e Questionários; Rede Social; Atividade Motora; Exercício.

\begin{abstract}
The physical activity has undergone changes with the advancement of internet. Gym users use this technology to connect to virtual social networks like Facebook. The purpose of this study is to develop and validate a questionnaire to use Facebook as a tool to investigate buman behavior in physical activity. From the theoretical framework was drawn up 20 items divided in five domains. The internal validation of the instrument was performed in five steps: a) Cronbach's alpha $(\alpha C)$; b) Kendall correlation; c) intraclass correlation coefficient; d) ceiling effects/floor; e) Kappa index. The sample consisted of 385 individuals, 273 women (70.9\%) with mean age 31.61 99.62. The values assigned to $\alpha \mathrm{C}$ coefficient demonstrate there is a excellent reliability of the questions (items) and answers $(\alpha C=0.923)$. By applying the correlation of $\alpha C\left(r^{2}\right)$ we found all the values above the expected: $\geq 0,3$ regarding that there is a positive association between items and domains. The values found in the application of the floor and ceiling effects show us that 10 items (50\%) had a prevalence of minimal response option (never). The reproducibility through Kappa index assigned maximum value (very good) to 11 items, and none was the weakest classification (poor). However, the validation showed that the instrument has a good performance to assess what is proposed.
\end{abstract}

\section{Keywords}

Surveys and Questionnaires; Social Networking; Motor Activity; Exercise.
1 Centro Universitário da Serra Gaúcha (FSG), Caxias do Sul, Rio Grande do Sul, Brasil. 2 Centro Universitário da Serra Gaúcha (FSG), Caxias do Sul, Rio Grande do Sul, Brasil e Pontifícia Universidade Católica do Rio Grande do Sul (PUCRS) Porto Alegre, Rio Grande do Sul, Brasil.

3 Universidade Federal do Rio Grande do Sul (UFRGS) Porto Alegre, Rio Grande do Sul, Brasil.

4 Centro Universitário da Serra Gaúcha (FSG), Caxias do Sul, Rio Grande do Sul, Brasil.

\section{Introdução}

O profissional de Educação Física necessita conhecer seu aluno para melhor motivá-lo à prática de atividades físicas (PAF), garantindo assim à promoção de um estivo de vida ativo e saudável ${ }^{1}$. A mídia colabora com esta promoção por meio da divulgação de ideias 
direcionadas ao combate do sedentarismo, influenciando os padrões estéticos que aumentam a demanda pela procura dos serviços disponibilizados pelas academias $^{2}$. As academias por sua vez, se tornam centros onde não há só o apelo estético, mas também a busca por socialização e a criação de vínculos que se externam a estes ambientes ${ }^{2}$.

As redes sociais virtuais permitem um convívio marcado pela comunicação instantânea e o aumento do número de contatos ${ }^{1}$. O Facebook é uma rede social virtual que permite o compartilhamento de sentimentos e experiências, bem como o acesso a novidades que influenciam nosso contexto ${ }^{3}$. Tornar públicas as imagens ou mídias em um site de rede social virtual se tornou uma prática social, onde ser notado pelo outro é decisivo para sua existência ${ }^{4}$. No Brasil, aproximadamente 65 milhões de pessoas acessam o Facebook diariamente. Destas, cerca de 50 milhões por meio de seus dispositivos móveis ${ }^{4}$.

A motivação por meio das redes sociais virtuais é uma interessante estratégia para a manutenção da prática de atividades físicas em universitários ${ }^{5}$. A internet tende a inspirar pessoas a terem um estilo de vida mais saudável por meio de páginas com imagens e textos que abordam este contexto. No entanto, a exposição aguda neste tipo de rede virtual influencia, sobre tudo, na imagem corporal dos praticantes, colocando-os em um estado de comparação ${ }^{6}$, podendo interferir negativamente no comportamento durante o desempenho das atividades propostas pelo instrutor da academia.

Contudo, alguns pesquisadores se preocupam no desenvolvimento e validação de instrumentos para mensurar a dependência em redes sociais online e a dependência pela conexão à internet em relação ao uso de redes sociais, como no caso do Facebook ${ }^{7-9}$. Dois destes instrumentos possuem validação para o idioma português ${ }^{10,11}$. O uso abusivo da internet pode ser um impacto negativo para o convívio social, o que justifica a criação de instrumentos de pesquisa para avaliação deste meio virtual ${ }^{12}$. No entanto, poucos estudos examinam a relação positiva das mídias virtuais com a promoção de atividades físicas, como no caso de Pumper $(2015)^{13}$, que avaliou a formação de grupos via Facebook em adolescentes que priorizassem a prática de exercícios físicos, sendo percebidas mudanças positivas tanto no comportamento social, quanto na prática de exercícios físicos regulares.

Assim, este estudo tem como objetivo desenvolver e validar um questionário sobre o uso do Facebook e a prática de atividades físicas nos contextos de socialização, comunicação, popularidade, entretenimento e conhecimento.

\section{Métodos}

\section{Construção do instrumento}

\section{- Referencial teórico}

Para construção do instrumento, inicialmente foi realizada uma busca para estruturação do referencial teórico, por meio de artigos científicos publicados a partir do ano de 2000, que abordassem especificamente o tema: redes sociais (Facebook) e a prática de atividades físicas. Também buscou-se localizar instrumentos validados que avaliassem tal contexto. Desta forma, foram selecionadas informações que colaborassem com a construção de questões capazes de relacionar o tema em questão (redes sociais e prática de atividades físicas). Considerando dois tópicos totalmente distintos, mas ao mesmo tempo tão proximais, onde o avanço das tecnologias no cotidiano das pessoas e a procura gradual pela PAF podem levar os adeptos às redes sociais a procurarem ou utilizarem tais recursos para aprimora- 
mento ou divulgação desta via social-digital.

Com o aumento da prática e utilização das redes sociais virtuais, percebe-se a criação de um relacionamento cibernético (online), com necessidade de atender as expectativas dos usuários, por meio de uma autorregulação, permitindo ocultar imagens indesejadas de si próprios e de suas vidas ${ }^{15}$. Além disso, um estudo ${ }^{16} \mathrm{com}$ plementa o contexto, dizendo que as mulheres possuem uma tendência em postar publicações relacionadas a sua massa corporal (peso), imagem corporal, exercício físico e dieta alimentar, passando a reparar em imagens e mensagens de amigos relacionados a tais aspectos de popularidade.

Apesar de o termo "social" estar ativamente relacionado as redes sociais virtuais, o uso excessivo do mesmo está também relacionado a fatores de personalidade e de solidão ${ }^{17}$. Há um uso mais intenso do Facebook para comunicação se comparado ao uso de outras redes como, por exemplo, o Twitter, sendo destacado que o conteúdo do Facebook está, em sua maior parte, relacionado ao entretenimento e conhecimento, sendo que a utilização de imagens está relacionada a um maior número de "curtidas"18.

\section{- Formulação do instrumento de coleta e avaliação}

O instrumento de coleta de dados foi baseado em uma escala de cinco pontos, no formato Likert ${ }^{14}$. Basicamente, esta escala consiste numa série de afirmações, em que o respondente deve expressar seu grau de frequência a cada afirmação, onde cada posição representa um valor numérico: 1) "nunca"; 2) "raramente"; 3) "às vezes”; 4) "frequentemente" e 5) "sempre”, com exceção do domínio Entretenimento (itens 13 a 16) que possuem escala Likert invertida: 5) "nunca"; 4) "raramente"; 3) "às vezes"; 2) "frequentemente" e 1) "sempre".

Esta escala apresenta uma série de vantagens, como: fornecer direções sobre a concepção do respondente em relação a cada item do instrumento e a não ambiguidade das categorias de respostas (por estarem previamente determinadas), evitando respostas que possam dificultar a interpretação por parte do avaliado ${ }^{14}$. Além disso, instrumento foi projetado com 20 itens (questões), divididas em cinco domínios: Socialização (itens: 1 a 4); Comunicação (itens: 5 a 8); Popularidade (itens: 9 a 12); Entretenimento (itens: 13 a 16); Conhecimento (itens: 17 a 20). Para avaliação do instrumento, optou-se pela somatória dos pontos (20 a 100), sendo codificada numa escala de 0 a 100 pontos, tendo como base para pontuação do escore total a fórmula simplificada [Total $=\left(\left(\left(\sum 20\right.\right.\right.$ itens $\left.\left.\left.)-20\right) \times 100\right) / 100\right]$. Além da pontuação total, o instrumento pode ser avaliado por domínios, sendo estratificada conforme fórmula [Módulo=((Média do módulo-1)/4)x100].

\section{Validação do instrumento}

\section{- Validade de conteúdo}

Para fins de validação de conteúdo, o questionário foi encaminhado a três pesquisadores da área de conhecimento, para análise da consistência de conteúdo e referenciais relacionados, sendo estruturado um formulário com três opções de ajustes: a) item de acordo com o tema, sem necessidades de ajuste ou exclusão; b) item de acordo com o tema, mas com necessidades de ajuste e c) item sem concordância com o tema, necessitando exclusão ou substituição. Dentre os 20 itens explorados, nenhum recebeu atribuição (c), sendo atribuídos apenas conceitos (a/b). Assim, as atribuições de conceitos (b) receberam ajustes quanto às sugestões dos pesquisadores ${ }^{19}$. Além disso, como sugestão dos avaliadores houve modificação no formato de perguntas abertas para itens pré-estruturados, sendo incorporada 
a escala de likert, para padronização das respostas e facilitação da análise do instrumento. Desta forma, a versão final do instrumento foi estabelecida em 20 itens, divididos em cinco domínios. Para validade fatorial dos domínios foi aplicado o teste de Kaiser-Meyer-Olkin (KMO) com níveis aceitáveis >0,6; teste de esfericidade de Bartlett's com níveis aceitáveis de $X^{2}$ para $\mathrm{p}<0,001$; e rotação fatorial Varimax com níveis aceitáveis $>0,6^{15}$.

\section{- Propriedade psicométrica}

As propriedades psicométricas estudadas foram confiabilidade e reprodutibilidade, sendo consideradas cinco análises a) alfa de Cronbach $(\alpha C)^{20}$ : que avalia se um instrumento é capaz de mensurar sempre da mesma forma o que se pretende medir, realizando uma correlação média entre perguntas e respostas. Assim, o coeficiente $\alpha C$ é calculado a partir da variância dos itens individuais e da variância da soma entre itens, verificando se todos utilizam a mesma escala de medição. Os valores foram considerados aceitáveis para pontuações de $\alpha$-C $>0,70$ e $<0,95$; b) correlação entre os itens categóricos, mediante a correlação de Kendall, considerando aceitáveis, valores $\mathrm{r} \geq 0,3^{21}$; c) coeficiente de correlação intraclasse $(\mathrm{CCI})^{21}$ : para mensurar a homogeneidade das medidas, servindo para interpretar a proporção da variabilidade total atribuída ao objeto medido, sendo adotado o ponto de corte $\geq 0,75$ para classificação do escore total (excelente); d) efeitos teto e piso $^{22}$ : que avaliam se considerados valores substanciais ultrapassassem $20 \%$ para as respostas ( $1=\mathrm{Nunca} /$ Piso e $5=$ Sempre/Teto); e) índice de $K a p p a^{23}$ : para avaliar a reprodutibilidade do instrumento em um determinado período do tempo (teste e reteste), sendo aplicado em pelo menos $10 \%$ dos participantes, com intervalo entre duas semanas. Para análise de concordância, valores $\leq 0,2$ são considerados pobres, entre 0,21-0,40, fracos, entre 0,41-0,60, moderados, entre 0,61-0,80, bons e $\geq 0,81$ muito bons, ou seja >6,0 são considerados aceitáveis.

\section{Participantes da pesquisa}

Participaram do estudo praticantes de atividades físicas, de ambos os sexos, com idade igual ou superior a 18 anos, devidamente matriculados em academias do município de Caxias do Sul/RS. Como critério de inclusão, os participantes deveriam estar matriculados ao menos na modalidade de musculação, com frequência mínima de duas vezes por semana, com duração $\geq 45$ minutos. Além disso, os participantes deveriam possuir conta na rede social Facebook.

\section{Coleta e análise dos dados}

O questionário foi aplicado na forma auto administrada, sendo respondido pelos próprios pesquisados, tendo os dados coletados armazenados em uma tabela no banco de dados Microsoft Access (Microsoft Corporation, Redmond, Washington, Estados Unidos), versão 2013, e exportados para o software estatístico SPSS (Statistical Package for the Social Sciences, Nova York, Estados Unidos) versão 20 para Windows. Foram realizados os seguintes testes: Critério de Kaiser-Meyer-Olkin (KMO) para análise da matriz de correlação inversa, teste de esfericidade de Bartlett's para testar as hipóteses correlacionadas e o teste de rotação Varimax para analisar as cargas fatoriais de cada domínio; coeficientes de $\alpha$ Cronback $(\alpha C)$, correlação do coeficiente intraclasse (ICC) e correlação de Spermann para fins de validade da consistência interna do instrumento e análise de Kappa para fins de reprodutibilidade. Além disso, os dados foram expressos por frequências absolutas e relativas para variáveis categóricas e média e desvio-padrão para variáveis contínuas. 


\section{Aspectos éticos}

O estudo foi aprovado pelo Comitê de Ética em Pesquisa (CEP) da instituição proponente sob o parecer consubstanciado $n^{\circ} 1.404 .497 / 2015$. Todos participantes assinaram um Termo de Consentimento Livre e Esclarecido (TCLE), além de uma anamnese com informações referentes à identificação, atividades físicas praticadas, motivação para a PAF, histórico de doenças/lesões e conectividade. O tempo estimado de preenchimento foi de dez minutos.

\section{Resultados}

Participaram do estudo 385 sujeitos, 273 do sexo feminino (70,9\%), com média de idade de 31,61 $\pm 9,62$ anos e com escolaridade predominante aos ensinos médio e superior ( $45,2 \%$ e $48,3 \%$, respectivamente). Do total, $73 \%$ praticam mais de uma modalidade esportiva na academia onde foram recrutados com frequência média de 4,26 $\pm 1,27$ (dia/semana) e tempo de 79,25 $\pm 32,01$ (minutos/dia). Apenas 53 (13,8\%) praticam atividades físicas por recomendações médica, tendo como motivação predominante o condicionamento físico (325, 84,4\%). Além disso, $279(72,5 \%)$ dos participantes utilizam ao menos duas redes sociais e $369(95,8 \%)$ utilizam as redes sociais via smartphone. Na avaliação do uso de redes sociais (Facebook) a pontuação média na utilização das mesmas para fins de atividade física foi de 44,70 $\pm 22,17$, numa escala de 0 a 100 pontos, onde quanto maior a pontuação, maior é a prática da utilização das redes sociais para fins de atividade física. Já na avaliação por domínios a pontuação mínima foi para o domínio popularidade $(24,79 \pm 24,31)$ e máxima para o domínio conhecimento $(54,94 \pm 21,08), \mathrm{p}<0,001$ (Tabela 1).

\section{Validade de conteúdo}

$\mathrm{Na}$ avaliação fatorial dos itens por domínio, o questionário apresentou valores dentro dos aceitáveis para os três testes aplicados, sendo: critério de Kaiser-Meyer-Olkin $(\mathrm{KMO}=0,887)$, teste de esfericidade de Bartlett's, demonstrando valores de $\mathrm{X}^{2}=\mathrm{p}<0,001$ e teste rotação Varimax, apresentando cinco fatores, com carga mínima -0,887 e máxima de 0,887, conforme tabela 2.

\section{Propriedade psicométrica}

\section{- Consistência interna}

Foi aplicado o $\alpha \mathrm{C}$ para a pontuação do escore total (20 itens), por domínios (5 domínios) e por item. $\mathrm{Na}$ análise de pontuação do escore total, o instrumento obteve valor de $\alpha C$ igual a 0,92 pontos e valores entre domínios mínimos de 0,92 (domínio entretenimento) e máximos de 0,93 (domínio conhecimento). Já para a análise por itens, os valores de $\alpha C$ foram de 0,92 para pontuação mínima e de 0,93 para pontuação máxima. Na avaliação do CCI, os valores apresentados para o escore total foram considerados excelentes, com CCI de 0,93 (IC95\%: 0,92-0,94; $\mathrm{p}<0,001)$, conforme demonstrado na tabela 3 .

\section{- Efeitos teto e piso}

$\mathrm{Na}$ avaliação dos efeitos teto e piso, dez (50\%) dos itens apresentaram valores superiores a $20 \%$ para o efeito piso (nunca) e apenas dois (10\%) para o efeito teto (sempre), conforme demonstrado na tabela 3 .

\section{- Correlação por itens e por escore médio total}

$\mathrm{Na}$ avaliação da correlação entre itens e pelo escore da pontuação total média (Tabela 4) apenas duas perguntas do questionário não apresentaram correlações integrais 
com os demais itens (questões 5 e 19). No entanto, na correlação entre itens e a pontuação média total, os valores demonstram possuir níveis de significância aceitáveis.

TABELA 1 - Características da amostra do estudo, Caxias do Sul, Brasil, 2016 ( $n=385)$.

\begin{tabular}{|c|c|c|c|c|}
\hline & $n$ & $\%$ & MD & $\pm \mathrm{DP}$ \\
\hline Sexo (feminino) & 273 & 70,9 & & \\
\hline Idade (anos) & & & 31,61 & $\pm 9,62$ \\
\hline \multicolumn{5}{|l|}{ Escolaridade } \\
\hline Ensino básico & 6 & 0,6 & & \\
\hline Ensino fundamental & 19 & 4,9 & & \\
\hline Ensino médio & 174 & 45,2 & & \\
\hline Ensino superior & 186 & 48,3 & & \\
\hline \multicolumn{5}{|l|}{ Atividades práticas (AP) } \\
\hline Apenas uma modalidade & 104 & 27,0 & & \\
\hline Duas modalidades & 135 & 35,1 & & \\
\hline Três modalidades & 92 & 23,9 & & \\
\hline Quatro modalidades & 25 & 6,5 & & \\
\hline Cinco modalidades & 29 & 7,5 & & \\
\hline Frequência média de AP (dias/semana) & & & 4,26 & $\pm 1,27$ \\
\hline Tempo médio de AP (minutos/dia) & & & 79,25 & $\pm 32,01$ \\
\hline \multicolumn{5}{|l|}{ Motivo da atividade prática } \\
\hline Orientação médica & 53 & 13,8 & & \\
\hline Condicionamento físico & 176 & 45,7 & & \\
\hline Bem-estar & 325 & 84,4 & & \\
\hline Lazer & 88 & 22,9 & & \\
\hline Restrições para atividade prática & 28 & 7,3 & & \\
\hline \multicolumn{5}{|l|}{ Utilização de redes sociais } \\
\hline Uma rede social (Facebook) & 106 & 27,5 & & \\
\hline Duas redes sociais & 181 & 47,0 & & \\
\hline Três redes sociais & 69 & 17,9 & & \\
\hline Quatro redes sociais & 25 & 6,5 & & \\
\hline Cinco redes sociais & 4 & 1,0 & & \\
\hline SmatPhone/Redes sociais & 369 & 95,8 & & \\
\hline \multicolumn{5}{|l|}{ Pontuação do questionário } \\
\hline Domínio socialização & & & 43,80 & $\pm 21,59$ \\
\hline Domínio comunicação & & & 46,75 & $\pm 22,68$ \\
\hline Domínio popularidade & & & 24,79 & $\pm 24,31$ \\
\hline Domínio entretenimento & & & 53,24 & $\pm 21,20$ \\
\hline Domínio conhecimento & & & 54,94 & $\pm 21,08$ \\
\hline Escore total do questionário & & & 44,70 & $\pm 22,17$ \\
\hline
\end{tabular}

n: frequência absoluta; \%: frequência relativa; MD: Média; „DP: Desvio padrão.

\section{- Reprodutibilidade}

O grau de consistência nas respostas para cada item sobre a administração repetida do questionário, com aplicação do índice de Kappa, obteve 95\% das perguntas classificadas com grau "moderado" (20,0\%), "bom" (20,0\%) ou "muito bom" $(55,5 \%)$ (Tabela 5). 
TABELA 2 - Análise fatorial do questionário sobre o uso do Facebook e a prática de atividades físicas nos contextos de socialização, comunicação, popularidade, entretenimento e conhecimento, Caxias do Sul, Brasil, 2016 ( $n=385)$.

\begin{tabular}{|c|c|c|c|c|c|c|}
\hline Domínios & Perguntas & Fator 1 & Fator 2 & Fator 3 & Fator 4 & Fator 5 \\
\hline \multirow[t]{4}{*}{ Popularidade } & P10 & 0,877 & & & & \\
\hline & P12 & 0,865 & & & & \\
\hline & P11 & 0,832 & & & & \\
\hline & P09 & 0,746 & & & & \\
\hline \multirow[t]{4}{*}{ Conhecimento } & P18 & & 0,875 & & & \\
\hline & P17 & & 0,820 & & & \\
\hline & P19 & & 0,782 & & & \\
\hline & P20 & & 0,612 & & & \\
\hline \multirow[t]{4}{*}{ Comunicação } & P05 & & & 0,764 & & \\
\hline & P08 & & & 0,763 & & \\
\hline & P07 & & & 0,736 & & \\
\hline & P06 & & & 0,604 & & \\
\hline \multirow[t]{4}{*}{ Socialização } & P02 & & & & 0,758 & \\
\hline & P01 & & & & 0,750 & \\
\hline & P03 & & & & 0,690 & \\
\hline & P04 & & & & 0,680 & \\
\hline \multirow[t]{4}{*}{ Entretenimento } & P14 & & & & & $-0,887$ \\
\hline & P15 & & & & & $-0,860$ \\
\hline & P13 & & & & & $-0,760$ \\
\hline & P16 & & & & & $-0,666$ \\
\hline
\end{tabular}

TABELA 3 - Frequências, médias, correlação e Alfa de Cronbach (aC) de respostas (por item), Caxias do Sul, Brasil, 2016 ( $n=385$ ).

\begin{tabular}{|c|c|c|c|c|c|c|c|c|}
\hline \multirow{2}{*}{ Perguntas/questionário } & Nunca & Raramente & Às vezes & Frequentemente & Sempre & \multirow{2}{*}{$\mathrm{MD} \pm \mathrm{DP}$} & \multirow{2}{*}{$r^{2}$} & \multirow{2}{*}{$\alpha \mathbf{C}$} \\
\hline & $n(\%)$ & $n(\%)$ & $n(\%)$ & $n(\%)$ & $n(\%)$ & & & \\
\hline Domínio socialização & - & - & - & - & - & $2,752 \pm 0,863$ & $0,724^{*}$ & 0,92 \\
\hline P01 & $19(4,9)$ & $73(19,0)$ & $171(44,4)$ & $84(21,8)$ & $38(9,9)$ & $3,127 \pm 0,993$ & $0,440^{*}$ & 0,93 \\
\hline P02 & $78(20,3) \neq$ & $106(27,5)$ & $115(29,9)$ & $60(15,6)$ & $26(6,8)$ & $2,610 \pm 1,168$ & $0,607^{*}$ & 0,93 \\
\hline P03 & $72(18,7)$ & $109(28,3)$ & $118(30,6)$ & $58(15,1)$ & $28(7,3)$ & $2,639 \pm 1,160$ & $0,517^{*}$ & 0,93 \\
\hline P04 & $107(27,8) \ddagger$ & $69(17,9)$ & $104(27,0)$ & $69(17,9)$ & $36(9,4)$ & $2,631 \pm 1,309$ & $0,465^{*}$ & 0,93 \\
\hline Domínio comunicação & - & - & - & - & - & $2,870 \pm 0,907$ & $0,680^{*}$ & 0,93 \\
\hline P05 & 150(39,0)‡ & $62(16,1)$ & $93(24,2)$ & $53(13,8)$ & $27(7,0)$ & $2,338 \pm 1,305$ & $0,389^{*}$ & 0,93 \\
\hline P06 & $22(5,7)$ & $26(6,8)$ & $83(21,6)$ & $129(33,5)$ & $125(32,5) \neq$ & $3,803 \pm 1,135$ & $0,527^{*}$ & 0,93 \\
\hline P07 & $106(27,5) \ddagger$ & $94(24,4)$ & $105(27,3)$ & $48(12,5)$ & $32(8,3)$ & $2,496 \pm 1,246$ & $0,453^{*}$ & 0,93 \\
\hline P08 & $86(22,3) \neq$ & $59(15,3)$ & $104(27,0)$ & $101(26,2)$ & $359(9,1)$ & $2,844 \pm 1,286$ & $0,483^{*}$ & 0,93 \\
\hline Domínio popularidade & - & - & - & - & - & $1,991 \pm 0,972$ & $0,695^{*}$ & 0,93 \\
\hline P09 & $218(56,6) \ddagger$ & $75(19,5)$ & $59(15,3)$ & $21(5,5)$ & $12(3,1)$ & $1,790 \pm 1,085$ & $0,588^{*}$ & 0,93 \\
\hline P10 & 171(44,4)‡ & $93(24,2)$ & $78(20,3)$ & $28(7,3)$ & $15(3,9)$ & $2,021 \pm 1,136$ & $0,539^{*}$ & 0,93 \\
\hline P11 & 193(50,1)‡ & $85(22,1)$ & $60(15,6)$ & $40(10,4)$ & $7(1,8)$ & $1,917 \pm 1,110$ & $0,518^{*}$ & 0,93 \\
\hline P12 & 167(43,4)‡ & $59(15,3)$ & $83(21,6)$ & $52(13,5)$ & $24(6,2)$ & $2,239 \pm 1,303$ & $0,598^{*}$ & 0,93 \\
\hline Domínio entretenimento & - & - & - & - & - & $3,083 \pm 0,916$ & $0,807^{*}$ & 0,92 \\
\hline
\end{tabular}

Continua... 
.... continua

\begin{tabular}{lcccccccc} 
P13 & $60(15,6)$ & $121(31,4)$ & $123(31,9)$ & $52(13,5)$ & $29(7,5)$ & $2,360 \pm 1,123$ & $0,621^{*}$ & 0,93 \\
P14 & $45(11,7)$ & $105(27,3)$ & $127(33,0)$ & $78(20,3)$ & $30(7,8)$ & $2,852 \pm 1,112$ & $0,609^{*}$ & 0,93 \\
P15 & $34(8,8)$ & $91(23,6)$ & $130(33,8)$ & $75(19,5)$ & $55(14,3)$ & $3,068 \pm 1,164$ & $0,696^{*}$ & 0,93 \\
P16 & $23(6,0)$ & $101(26,2)$ & $132(34,3)$ & $77(20,0)$ & $52(13,5)$ & $3,088 \pm 1,112$ & $0,602^{*}$ & 0,93 \\
Domínio conhecimento & - & - & - & - & - & $3,197 \pm 0,843$ & $0,658^{*}$ & 0,93 \\
P17 & $35(9,1)$ & $85(22,1)$ & $112(29,1)$ & $108(28,1)$ & $45(11,7)$ & $3,112 \pm 1,150$ & $0,465^{*}$ & 0,93 \\
P18 & $12(3,1)$ & $42(10,9)$ & $130(33,8)$ & $143(37,1)$ & $58(15,1)$ & $3,501 \pm 0,979$ & $0,547^{*}$ & 0,93 \\
P19 & $20(5,2)$ & $48(12,5)$ & $100(26,0)$ & $132(34,3)$ & $85(22,1) \neq$ & $3,556 \pm 1,119$ & $0,336^{*}$ & 0,93 \\
P20 & $101(26,2) \neq$ & $80(20,8)$ & $106(27,5)$ & $60(15,6)$ & $38(9,9)$ & $2,621 \pm 1,292$ & $0,489^{*}$ & 0,93 \\
Média escore total & - & - & - & - & - & $2,779 \pm 0,664$ & $0,891^{*}$ & 0,92 \\
\hline
\end{tabular}

n: frequência absoluta; \%: frequência relativa; MD士DP: Média e desvio padrão; aC: Coeficiente de Alfa de Cronbach; r2: correlação do aC; * $p$-valor de $r 2<0,05$; $\neq$ : efeito piso/teto para itens com prevalência acima de $20 \%$ das respostas.

TABELA 4 - Correlação por item e pelo total da pontuação das 20 perguntas compostas pelo questionário, Caxias do Sul, Brasil, 2016 ( $n=385)$.

\begin{tabular}{|c|c|c|c|c|c|c|c|c|c|c|c|c|c|c|c|c|c|c|c|c|c|}
\hline & P1 & P2 & P3 & P4 & P5 & P6 & P7 & P8 & P9 & P10 & P11 & P12 & P13 & P14 & P15 & P16 & P17 & P18 & P19 & P20 & ET \\
\hline P1 & 1,000 & $0,491^{* *}$ & $0,328^{* *}$ & $0,343^{* *}$ & 0,091 & $0,248^{* *}$ & $0,249^{* *}$ & $0,143^{* *}$ & $0,228^{* *}$ & $0,267 * *$ & $0,189^{* *}$ & $0,265 *$ & * $0,199 *$ & * $0,206 *$ & * $0,178 * *$ & * $0,294 *$ & $0,193^{* *}$ & $0,206 * *$ & 0,104 & $0,338^{* *}$ & $0,464 * *$ \\
\hline P2 & & 1,000 & $0,489^{* *}$ & $0,437^{* *}$ & $0,176^{* *}$ & ${ }^{*} 0,368^{* * *}$ & $0,234^{* *}$ & $0,208^{* * *}$ & $0,397^{* *}$ & $0,392^{* * *}$ & $0,313^{* *}$ & 0,429 & $0,297 \ldots *$ & * 0,334** & * 0,420 & * $0,502 * *$ & $0,236^{\text {*** }}$ & $0,278 *$ & $0,135 * *$ & $0,340^{* * *}$ & $0,650^{* *}$ \\
\hline P3 & & & 1,000 & $0,403^{* *}$ & $0,148^{* *}$ & ${ }^{*} 0,281^{* *}$ & $0,218^{* *}$ & $0,231^{* *}$ & $0,337^{* *}$ & $0,324 * *$ & $0,267^{* *}$ & * $0,310^{* * *}$ & $0,248 * *$ & * $0,341 * *$ & * $0,329 * *$ & * $0,370^{* *}$ & $0,219 * *$ & $0,224 * *$ & $0,111^{*}$ & $0,380^{* *}$ & $0,563 * *$ \\
\hline P4 & & & & 1,000 & $0,113^{*}$ & $0,239^{* * *}$ & $0,163^{* *}$ & $0,172^{* *}$ & $0,274^{* *}$ & $0,321 * *$ & $0,216^{* *}$ & 0,349 & 0,256 & * $0,250 * *$ & * $0,280 * *$ & * 0,349*** & $0,185^{\text {** }}$ & $0,207 * *$ & $0,117 *$ & $0,338^{* *}$ & $0,537_{* *}$ \\
\hline P5 & & & & & 1,000 & $0,268^{* *}$ & $0,389^{* *}$ & $0,429^{* *}$ & $0,282^{* *}$ & $0,159 * *$ & $0,288^{* *}$ & $0,201^{* *}$ & $0,217 * *$ & $* 0,253 * *$ & $* 0,262 *$ & * $0,288^{* *}$ & $0,131^{* *}$ & $0,162^{* *}$ & 0,068 & $0,158^{* *}$ & $0,442 * *$ \\
\hline P6 & & & & & & 1,000 & $0,330^{* *}$ & $0,391^{* *}$ & $0,297^{* *}$ & $0,244^{* * *}$ & $0,284^{* *}$ & $0,333^{* * *}$ & $0,380^{* * *}$ & * 0,336** & * $0,278 * *$ & $* 0,365 * *$ & $0,205^{\text {*** }}$ & $0,239 * *$ & $0,108^{*}$ & $0,227^{* * *}$ & $0,556 * *$ \\
\hline P7 & & & & & & & 1,000 & $0,390^{* *}$ & $0,260^{* *}$ & 0,130 & $0,176^{* *}$ & $0,202^{* *}$ & $0,293 * *$ & $* 0,322 *$ & $* 0,289 *$ & * $0,268 *$ & $0,157 * *$ & $0,216 * *$ & 0,099 & $0,261 * *$ & 0,491 ** \\
\hline P8 & & & & & & & & 1,000 & $0,306^{* *}$ & $0,194 * *$ & $0,242^{* *}$ & ${ }^{*} 0,222^{* *}$ & 0,279 ** & * $0,321 * *$ & * $0,280 * *$ & * $0,346^{* *}$ & $0,191_{* *}$ & 0,269 ** & $0,188^{* *}$ & * $0,233^{* *}$ & $0,533^{* * *}$ \\
\hline P9 & & & & & & & & & 1,000 & $0,538 * *$ & $0,518^{* *}$ & $0,537^{* *}$ & $0,336 * *$ & * $0,297 * *$ & * $0,386 * *$ & * 0,490 & $0,210^{* * *}$ & $0,193 *$ & 0,049 & $0,223^{* *}$ & $0,622^{* *}$ \\
\hline P10 & & & & & & & & & & 1,000 & $0,595^{* *}$ & $0,763^{* *}$ & "0,302** & * $0,240^{* * *}$ & * $0,300 * *$ & * $0,384 * *$ & $0,150^{* * *}$ & $0,143 * *$ & 0,021 & 0,236 ** & $0,589 * *$ \\
\hline P11 & & & & & & & & & & & 1,000 & $0,681^{* * *}$ & 0,309 w* & * $0,235 * *$ & * $0,283 * *$ & * $0,408 * *$ & $0,104^{*}$ & $0,133 * *$ & 0,004 & 0,199 & $0,558 * *$ \\
\hline P12 & & & & & & & & & & & & 1,000 & $0,363 * *$ & * $0,266 * *$ & $* 0,332 * *$ & $* 0,463 * *$ & $0,182^{* *}$ & $0,196 * *$ & 0,051 & $0,281 * *$ & $0,653^{* *}$ \\
\hline P13 & & & & & & & & & & & & & 1,000 & $0,637 * *$ & * $0,509 * *$ & * $0,430^{* *}$ & $0,361 * *$ & $0,482^{* *}$ & * 0,269** & $0,258^{* *}$ & $0,641_{* *}$ \\
\hline P14 & & & & & & & & & & & & & & 1,000 & 0,680 & $* 0,496 *$ & $0,339^{* * *}$ & $0,404 * *$ & $0,254 *$ & $0,313^{* * *}$ & $0,652^{* * *}$ \\
\hline P15 & & & & & & & & & & & & & & & 1,000 & $0,592 * *$ & $0,326 * *$ & $0,409 * *$ & $0,256 * *$ & * 0,339** & $0,679 * *$ \\
\hline P16 & & & & & & & & & & & & & & & & 1,000 & $0,377_{* * *}$ & $0,384 * *$ & * $0,243 * *$ & $0,342^{* *}$ & $0,727 * *$ \\
\hline P17 & & & & & & & & & & & & & & & & & 1,000 & $0,693^{* *}$ & * $0,394 * *$ & $0,269 * *$ & $0,500^{* * *}$ \\
\hline P18 & & & & & & & & & & & & & & & & & & 1,000 & $0,512^{* * *}$ & $0,323 * *$ & $0,565 * *$ \\
\hline P19 & & & & & & & & & & & & & & & & & & & 1,000 & $0,274 *$ & $0,359 * *$ \\
\hline P20 & & & & & & & & & & & & & & & & & & & & 1,000 & $0,552^{* *}$ \\
\hline
\end{tabular}

P: Pergunta correspondente ao número do questionário; ET: Escore total do questionário; **: p de $r<0,01$; *: p de $r<0,05$ a 0,01; Para avaliação categórica foi aplicada a correlação de Kendall (entre itens) e comparação entre os itens e o escore médio total foi aplicada a correlação de Spermann.

TABELA 5 - Reteste aplicado a 74 participantes duas semanas após a primeira avaliação do questionário sobre o uso do Facebook e a prática de atividades físicas, Caxias do Sul, Brasil, 2016 ( $n=385$ ).

\begin{tabular}{lccc}
\hline Grau & Kappa & $\%$ & Número do item de referência \\
\hline Pobre & $\leq 0,2$ & 0,0 & - \\
Fraco & $0,21-0,40$ & 5,0 & 05 \\
Moderado & $0,41-0,60$ & 20,0 & $01,02,03$ e 17 \\
Bom & $0,61-0,80$ & 20,0 & $04,08,09$ e 12 \\
Muito bom & $\geq 0,81$ & 55,0 & $06,07,10,11,13,14,15,16,18,19$ e 20 \\
\hline
\end{tabular}




\section{Discussão}

O desenvolvimento de um instrumento específico para avaliar o uso de ferramentas sociais virtuais, como no caso do Facebook para fins de conhecimento ou divulgação da importância das práticas esportivas é de extrema importância no contexto atual da sociedade. $\mathrm{O}$ uso de ferramentas virtuais para facilitar a vida diária das pessoas é cada vez mais explorado, abrindo margem para a doença crônica mais comum desta geração digital, o sedentarismo. Ao mesmo tempo, se tais ferramentas digitais forem aplicadas para divulgação ou autoconhecimento sobre a importância da PAF regular para manutenção e/ou melhora da saúde da sociedade, pode ter um papel fundamental para incentivo da prática. Assim, o presente estudo, teve como objetivo central o desenvolvimento e validação de um instrumento específico para avaliar o uso do Facebook para socialização, comunicação, popularidade, entretenimento e conhecimento de assuntos relacionados à prática de atividades físicas, demonstrando possuir excelente consistência interna e reprodutibilidade.

Na avaliação da validade de conteúdo do questionário, por meio da análise fatorial dos itens por domínio, os resultados apresentaram valores dentro dos padrões aceitáveis para os três testes aplicados, demonstrando uma boa correlação fatorial entre os 20 itens e os cinco domínios propostos $\left(\mathrm{KMO}=0,887\right.$, Bartlett $^{\prime} s=\mathrm{p}<0,001 \mathrm{e}$ rotação Varimax mínima de $-0,887$ e máxima de 0,887$)$. A validade da consistência interna por meio do Alfa de Chronbah $(\alpha \mathrm{C})$ demonstra que a aplicação de tal coeficiente nos permite avaliar a consistência entre itens ${ }^{24}$, bem como suas respostas, fornecendo uma estimativa da verdadeira fiabilidade de um instrumento para as devidas medições ${ }^{25}$, onde valores abaixo de 0,70 são considerados fracos e acima de 0,95 considerados redundantes. Assim, os valores atribuídos ao coeficiente de $\alpha C$ neste estudo demonstram existir excelente fiabilidade entre perguntas (itens) e suas respostas no formato escalar de likert $(\alpha C=0,92)$. Ao aplicarmos a correlação do $\alpha \mathrm{C}\left(\mathrm{r}^{2}\right)$ encontramos todos os valores acima do esperado: $\geq 0,3$ reforçando que há uma associação positiva entre itens e categorias.

$\mathrm{Na}$ validação do instrumento Bergen Facebook Addiction Scale $(\mathrm{BFAS})^{9}$, relacionado ao vício no Facebook e aplicado em 423 estudantes, foi obtido um $\alpha \mathrm{C}$ de $0,83 \mathrm{e}$ um coeficiente teste-reteste de 0,82 . Ambos os valores mais fracos que os obtidos pelo questionário do nosso estudo. Já a versão em português da escala da BFAS ${ }^{11}$, obteve $\alpha \mathrm{C}$ de 0,92 e CCI de 0,81, em uma amostra de 359 estudantes de graduação, pouco menor que o número de participantes neste estudo.

Outro instrumento relacionado ao uso da internet é o Internet Addiction Test $(\mathrm{IAT})^{8}$ que validou a confiabilidade de suas 20 questões com a aplicação em 86 usuários de internet e apresentou um $\alpha \mathrm{C}$ de 0,83 , o que lhe atribui ser um instrumento válido e confiável. A versão em português do IAT $^{10}$ apresentou $\alpha C$ de 0,84 (satisfatório) ao ser aplicado em uma amostra de 117 estudantes universitários, porém não foi aplicado o teste de reprodutibilidade, análise na qual nosso instrumento obteve bons resultados. Outro autor ${ }^{26}$ aplicou o IAT em 267 jovens com idade entre 12 e 17 anos de Santa Catarina/RS, obtendo $\alpha \mathrm{C}$ de 0,88, atingindo valores adequados para sua aplicação com adolescentes.

Os instrumentos BFAS e IAT são os instrumentos validados mais próximos ao contexto proposto pelo presente estudo, por estarem avaliando, sobretudo algum tipo de interação com a internet. No entanto, os aspectos abordados são distintos ao proposto pelo presente estudo em validação. Além dos instrumentos BFAS e IAT, não foram encontrados outros instrumentos que avaliem o envolvimento de praticantes de atividades físicas com redes sociais virtuais, embora alguns estudos 
ressaltem a necessidade de novas pesquisas para explorarem tais relações ${ }^{15,16}$.

Além disso, neste estudo foram aplicadas avaliações complementares as analisadas pelos dois instrumentos (BFAS e IAT), como no caso do efeito teto e piso, sendo considerada uma importante avaliação no processo de validação de instrumentos que utilizam respostas no formato likert, avaliando quanto as alternativas mínimas ou máximas influenciam nas respostas dos avaliados. Os valores encontrados com a aplicação dos efeitos de teto/piso nos mostram que 10 itens (50\%) obtiveram prevalência da opção de resposta mínima (nunca) o que acabou influenciando na média do total do questionário $44,47 \pm 16,62$. Talvez uma amostra com adultos jovens pudesse indicar uma maior interação, tendo em vista o estudo de Assunção (2014) ${ }^{27}$ que aponta que jovens relatam ver amigos transformarem o Facebook em um diário de sua vida.

Outro processo de validação importante aplicado neste estudo foi o de reprodutibilidade pelo índice de Kappa. Esta análise tem sido reportada por autores como ponto positivo nos processos de desenvolvimento e validação de instrumentos para avaliar preenchimentos aleatórios ou ao acaso, tanto para processos intervencionais (pré e pós intervenção) ou sem intervenção, respeitando prazo mínimo de uma semana e máximo de quatro semanas ${ }^{28,29}$. No presente instrumento a reprodutibilidade atribuiu valor máximo (muito bom) para 11 dos 20 itens submetidos, sendo que apenas a questão 5 foi classificada como fraca. Um estudo de $2012^{29}$ obteve reprodutibilidade moderada na validação de um instrumento que relaciona motivação com a prática de atividades físicas em seu instrumento que contava com 40 itens. Mesmo assim o instrumento tornou-se viável ao que se propõem.

Como principal limitação do presente estudo apontamos o elevado percentual de respostas para o efeito piso, onde $50 \%(\mathrm{n}=10)$ dos itens avaliados receberam mais de $20 \%$ nas respostas mínima (nunca), podendo ser um indicador de má formulação das respostas, ou em virtude da população estudada possuir um perfil de baixa procura ou utilização de redes sociais para a PAF. Ao mesmo tempo a literatura $^{30}$ aborda o quanto os questionários qualificam uma pesquisa e colaboram com a comunidade cientifica envolvida, sendo que a elaboração dos mesmos pode ser aprimorada com o tempo e com a aplicação em diferentes públicos.

Por fim, os valores obtidos demonstram que este instrumento é valido para o que se propõe e pode ser aplicado com alunos praticantes de atividades físicas em academias. Com este questionário, pesquisadores ou gestores de academia poderão adquirir informações precisas sobre o perfil de praticantes de atividades físicas regulares em academias e o uso de redes sociais para conhecimento ou convívio social direcionado a prática de exercícios, nos qual seus resultados podem mudar de acordo com o perfil do público da academia ou região na qual está inserida.

\section{Financiamentos}

Os recursos financeiros do estudo foram pagos pelos próprios pesquisadores, sem auxílio de agências de fomento ou patrocínios externos.

\section{Contribuição dos autores}

M. Pedroso (0000-0002-0778-7606) participou da elaboração do projeto, coleta de dados, análise estatística e escrita do artigo. R.F. Sartori (0000-0001-8093-6906) participou da elaboração do projeto e revisão crítica do artigo. C.P. Dias (00000002-8480-7527) participou da elaboração do projeto e revisão crítica do artigo. C; Roncada (0000-0003-3782-4911) participou da elaboração do projeto, análise estatística, escrita do artigo e revisão final. 


\section{Referencias}

1. Liz CMd, Crocetta TB, Viana $\mathrm{MdS}$, Brandt $\mathrm{R}$, Andrade A. Aderência à prática de exercícios físicos em academias de ginástica. Motriz. 2010;16(1):181-8.

2. Moreira JA. Motivos que levam as pessoas a praticarem exercícios em uma academia de musculação: um estudo de caso [trabalho de conclusão de curso - Bacharelado em Educação Física]. Criciuma/SC: Universidade Estadual de Santa Catarina. 2015.

3. Reis M, Cunha L, Marques A, Quintela RB, Brito JC, Rrodrigues L. O outro lado do facebook.. In: XI Congresso de Ciências da Comunicação na Região Norte [online]; 2012 Set 17-19; Palmas. Anais eletrônicos. Palmas: Sociedade Brasileira de Estudos Interdisciplinares da Comunicação; 2012. [Citado 2012 set 19]. Disponível em: http:// www.posemcomunicacaodigital.com.br/wp-content/files/biblioteca/OOutroLadodo Facebook.pdf.

4. Juliani DP, Juliani JP, de Souza JA, de Bettio RW. Utilização das redes sociais na educação: guia para o uso do Facebook em uma instituição de ensino superior. Renote. 2012;10(3)1-11.

5. Al-Eisa E, Al-Rushud A, Alghadir A, Anwer S, Al-Harbi B, Al-Sughaier N, et al. Effect of Motivation by "Instagram" on Adherence to Physical Activity among Female College Students. BioMed Research International, 2016;16.

6. Tiggemann M, Zaccardo M. "Exercise to be fit, not skinny": The effect of fitspiration imagery on women's body image. Body image. 2015;15:61-7.

7. Thadani DR, Cheung CM, editors. Online social network dependency: Theoretical development and testing of competing models. System Sciences (HICSS), 2011 44th Hawaii International Conference on; 2011: IEEE.

8. Widyanto L, McMurran M. The psychometric properties of the internet addiction test. Cyber Psychol Beh. 2004;7(4):443-50.

9. Andreassen CS, Torsheim T, Brunborg GS, Pallesen S. Development of a Facebook addiction scale 1, 2. Psych. Rep. 2012;110 (2):501-17.

10. Conti MA, Jardim AP, Hearst N, CORDáS TA, Tavares H, Abreu CNd. Avaliação da equivalência semântica e consistência interna de uma versão em português do Internet Addiction Test (IAT). Rev Psiq Clín. 2012;39(3):106-10.

11. Silva, HRDS. Areco, KCN. Bandiera-Paiva, P. Galvão, PVM. Garcia, ANDM, Silveira, DXD. Semantic equivalence and confiability of the Portuguese version of the Bergen Facebook Addiction Scale. J Bras Psiquiatria. 2015;64(1):17-23.

12. Abreu C, Karam RG, Góes DS, Spritzer DT. Dependência de Internet e de jogos eletrônicos: Uma revisão. Rev Bras Psiquiatria. 2008;30(2):156-67.

13. Pumper M, Mendoza J, Arseniev-Koehler A, Holm M, Wait A, Moreno M. Using a Facebook Group as an Adjunct to a Pilot Health Physical Activity Intervention: A Mixed Methods Approach. Annu Rev Cyberth Telemed. 2015;219:97-101.

14. Likert R. The method of constructing and attitude scale. Meth Techn Business Res. $1967 ; 54: 90-5$

15. Marder B, Houghton D, Joinson A, Shankar A, Bull E. Understanding the Psychological Process of Avoidance-Based Self-Regulation on Facebook. Cyberp Beh Social Netw. 2016;19(5):321-7.

16. Eckler P, Petya, Kalyango Jr P, Yusuf, Paasch M, Ellen. Facebook use and negative body image among US college women. Women Health. 2016;11(2):1-19.

17. Petrocchi N, Asnaani A, Martinez AP, Nadkarni A, Hofmann SG. Differences between people who use only Facebook and those who use Facebook plus Twitter. Inter J HumanComputer Inter. 2015;31(2):157-65.

18. Zago GDS, Bastos MT. Visibilidade de notícias no twitter e no facebook. Brazilian J Res. 2013;9(1):116-33.

19. Alexandre NMC, Coluci MZO. Validade de conteúdo nos processos de construção e adaptação de instrumentos de medidas.[Content validity in the development and adaptation processes of measurement instruments]. Ciência \& Saúde Col. 2011;16(7):3061-8.

20. Cronbach LJ. Coefficient alpha and the internal structure of tests. Psychometrika. 1951;16(3):297-334.

21. Bartko JJ. The intraclass correlation coefficient as a measure of reliability. Psychological reports. 1966;19(1):3-11.

22. Youngstedt SD. Ceiling and floor effects in sleep research. Sleep Medic Rev. 2003;7(4):351-65. 
23. Ullibarri Galparsoro L, Pita Fernández S. Medidas de concordancia: el índice de Kappa. Cad Aten Primaria. 1999;6:169-71.

24. Hora HRM, Monteiro GTR, Arica J. Confiabilidade em questionários para qualidade: um estudo com o Coeficiente Alfa de Cronbach. Produto Produção. 2010;11(2):85-103.

25. Maroco J, Garcia-Marques T. Qual a fiabilidade do alfa de Cronbach? Questões antigas e soluções modernas? Laborat Psicologia. 2013;4(1):65-90.

26. Beza TB, de Oliveira Wolff L, Basso F, Laurentino IC, Farias CAM, Philippi D. Correlação dos itens do Internet Addiction Test em uma amostra de jovens de 12 a 17 anos no Sul do estado de Santa Catarina. Rev Electr Investiga Docencia (REID). 2016;15:71-84.

27. Assunção RS, Matos PM. Perspetivas dos adolescentes sobre o uso do Facebook: um estudo qualitativo. Psicol Estud. 2014; 19(3):539-47.

28. Farias Júnior JCD, Lopes ADS, Mota J, Santos MP, Ribeiro JC, Hallal PC. Validade e reprodutibilidade de um questionário para medida de atividade física em adolescentes: uma adaptação do Self-Administered Physical Activity Checklist. Rev Bras Epidemiol. 2012;15(1):198-210.

29. Guedes DP, Lopes CC, Guedes J. Reprodutibilidade e validade do Questionário Internacional de Atividade Física em adolescentes. Rev Bras Med Esporte. 2005;11(2):151-8.

30. Vilas Boas, AVDO, Neto AMS. Elaboração e validação de questionário de avaliação da qualidade científica de pesquisas epidemiológicas. Rev Baiana Saúde Públ. 2012;36(2):432-64.

\section{Endereço para}

Correspondência

Cristian Roncada

crisron@gmail.com
Rua Os Dezoito do Forte, 2366 - São

Pelegrino, Caxias do Sul - RS, CEP

95020-472.

Telefone/fax: (54) 2101-6000.

$\begin{array}{ll}\text { Recebido } & 29 / 11 / 2016 \\ \text { Revisado } & 17 / 03 / 2017 \\ & 31 / 03 / 2017 \\ & 05 / 05 / 2017 \\ & 16 / 06 / 2017 \\ \text { Aprovado } & 18 / 12 / 2015\end{array}$




\section{Anexo 1}

\section{Questionário sobre uso do Facebook voltado para praticantes de atividades físicas em} academias.

\begin{tabular}{|c|c|c|c|c|c|}
\hline AFIRMAÇÃO & Nunca & Raramente & Às vezes & Frequentemente & Sempre \\
\hline $\begin{array}{l}1 \text { - Envia/recebe solicitações de amizade de amigos, conhecidos e profissionais da } \\
\text { academia. }\end{array}$ & $\square$ & $\square$ & $\square$ & $\square$ & $\square$ \\
\hline 2 - Compartilha mídias no Facebook com os seus amigos de academia. & $\square$ & $\square$ & $\square$ & $\square$ & $\square$ \\
\hline $\begin{array}{l}3 \text { - Conversa com os seus amigos, na academia, a respeito do conteúdo que você } \\
\text { visualizou no Facebook. }\end{array}$ & $\square$ & $\square$ & $\square$ & $\square$ & $\square$ \\
\hline 4 - Participa de grupos no Facebook formados a partir de sua convivência na academia. & $\square$ & $\square$ & $\square$ & $\square$ & $\square$ \\
\hline 5 - Entrou em contato com alguma academia através de sua página no Facebook. & $\square$ & $\square$ & $\square$ & $\square$ & $\square$ \\
\hline 6 - Acompanha publicações no Facebook da academia que você é aluno (a). & $\square$ & $\square$ & $\square$ & $\square$ & $\square$ \\
\hline 7 - Acompanha as publicações de academias que você nunca se matriculou. & $\square$ & $\square$ & $\square$ & $\square$ & $\square$ \\
\hline 8 - Conheceu uma academia ou modalidade através do Facebook. & $\square$ & $\square$ & $\square$ & $\square$ & $\square$ \\
\hline $\begin{array}{l}9 \text { - Compartilha em sua página as expectativas e/ou desilusões e/ou resultados obtidos } \\
\text { com a academia. }\end{array}$ & $\square$ & $\square$ & $\square$ & $\square$ & $\square$ \\
\hline $\begin{array}{l}10 \text { - Compartilha imagens sua, sozinho (a) ou acompanhado (a), capturadas enquanto } \\
\text { estava na academia. }\end{array}$ & $\square$ & $\square$ & $\square$ & $\square$ & $\square$ \\
\hline 11 - Publica no Facebook que você está na academia. & $\square$ & $\square$ & $\square$ & $\square$ & $\square$ \\
\hline $\begin{array}{l}12 \text { - Recebe um considerável número de "curtidas" em suas imagens capturadas } \\
\text { enquanto estava na academia. }\end{array}$ & $\square$ & $\square$ & $\square$ & $\square$ & $\square$ \\
\hline 13 - Visualiza conteúdo motivacional relacionado a pratica de atividade física. & $\square$ & $\square$ & $\square$ & $\square$ & $\square$ \\
\hline 14 - Visualiza conteúdo de humor relacionado a pratica de atividade física e academias. & $\square$ & $\square$ & $\square$ & $\square$ & $\square$ \\
\hline $\begin{array}{l}15 \text { - Curte e/ou compartilha conteúdos de humor ou motivação relacionados a pratica } \\
\text { de atividade física ou academia. }\end{array}$ & $\square$ & $\square$ & $\square$ & $\square$ & $\square$ \\
\hline $\begin{array}{l}16 \text { - Interage (curte, compartilha, marca amigos) com as publicações do Facebook que } \\
\text { estão relacionadas com a academia ou atividade física. }\end{array}$ & $\square$ & $\square$ & $\square$ & $\square$ & $\square$ \\
\hline $\begin{array}{l}\text { 17- Visualiza conteúdo acadêmico/científico relacionado à Educação Física e demais } \\
\text { cursos da área da saúde. }\end{array}$ & $\square$ & $\square$ & $\square$ & $\square$ & $\square$ \\
\hline 18 - Visualiza conteúdo informativo relacionado à atividade física. & $\square$ & $\square$ & $\square$ & $\square$ & $\square$ \\
\hline 19 - Verifica a confiabilidade das informações relacionadas à atividade física e saúde. & $\square$ & $\square$ & $\square$ & $\square$ & $\square$ \\
\hline $\begin{array}{l}20 \text { - Conversa ou esclarece dúvidas com seu instrutor a respeito de conteúdos } \\
\text { visualizados no Facebook. }\end{array}$ & $\square$ & $\square$ & $\square$ & $\square$ & $\square$ \\
\hline
\end{tabular}




\section{Anexo 2}

\section{Instruções, pontuação e cálculo para o questionário.}

\section{- Instruções}

Caro pesquisador, solicite ao respondente que responda os 20 itens utilizando apenas uma das opções de resposta (nunca, raramente, às vezes, frequentemente ou sempre) deixando claro que todas questões são referentes unicamente ao uso da rede social Facebook.

\section{- Pontuação para as respostas:}

Perguntas (1 a 20):

Nunca $=1$ ponto;

Raramente $=2$ pontos;

Às vezes $=3$ pontos;

Frequentemente $=4$ pontos;

Sempre $=5$ pontos.

Importante: As Questões 13, 14, 15 e 16 possuem valor inverso ou seja: nunca $=5$ pontos, raramente $=4$ pontos, às vezes $=3$ pontos, frequentemente $=2$ pontos e sempre $=1$ ponto.

Cálculo da pontuação média dos itens (valores expressos de 1 a 5 pontos):

$$
\text { Média }=(\text { soma dos escores das } 20 \text { perguntas }) \div 20 \text {. }
$$

Média: Transformação da soma total dos itens em média de 1 a 5

\section{- Cálculo da pontuação do item total (valores expressos de 0 a 100 pontos):}

$$
\text { Total }=(\text { Média })-1 \times 100 \div 4
$$

Total: Transformação das médias de 1 a 5, para 0 a 100 :

- 1: Menor possibilidade de resposta (1-1:0)

4: Possibilidade do valor bruto por itens (5-1: 4)

100: Transformação da estala de 0 a 4 para 0 a 100

$\mathrm{Ou}$

\section{- Cálculo direto (valores expressos de 0 a 100 pontos):}

$$
\text { Total }=(\text { Soma dos } 20 \text { itens })-20 \times 100 \div 100
$$

Total: Transformação das médias de 1 a 5, para 0 a 100:

- 20: Menor possibilidade de resposta [( $1 * 20)-20$ : 0]

100: Possibilidade do valor bruto por itens [(5*20)-20: 100]

100: Transformação da estala de 0 a 4 para 0 a 100 